\title{
TOWARD AN ACCURATE MULTI-FIBER ASSESSMENT STRATEGY FOR CLINICAL PRACTICE.
}

\author{
Benoit Scherrer, Simon K. Warfield \\ Computational Radiology Laboratory, Department of Radiology \\ Children's Hospital, 300 Longwood Avenue, Boston, MA, 02115, USA
}

\begin{abstract}
Multi-tensor models provide information about the fiber bundles underlying characteristics and are of great interest for clinical applications. In this work we propose both a novel model fitting procedure and a novel acquisition scheme for multi-tensor assessment. Our fitting procedure includes the estimation of the diffusion of free water and ensures nondegenerate and regularized tensors. Our acquisition scheme combines spherical and cubic sampling. It enables the acquisition of multiple non-zero b-values which has been shown to be required for the full multi-tensor estimation. It enables high b-values to be acquired while achieving the same low echo time as a single-shell HARDI, leading to comparable geometrical and intensity distortion. We evaluate our resulting novel strategy CUSP-MFM (CUbe+SPhere Multi-Fiber Model) on both synthetic and clinical data. We show the ability of CUSPMFM of assessing complex fiber structures from short duration acquisitions, compatible with clinical routine.
\end{abstract}

Index Terms - diffusion imaging, multi-tensor fitting, multiple b-values acquisition scheme.

\section{INTRODUCTION}

A large number of approaches have been proposed to overcome the limitations of Diffusion Tensor Imaging (DTI) [1] which cannot represent complex fiber structures. They involve both novel diffusion signal sampling schemes and novel ways to analyse the diffusion signal. Three major sampling schemes have been proposed for complex structure assessment: cartesian sampling, single-shell and multiple-shell spherical sampling (high angular resolution diffusion imaging, HARDI). Various model-free and model-based approaches have been investigated to analyse the diffusion-weighted (DW) signal. Most of them focus on describing the general shape of the diffusion profile in each voxel: diffusion spectrum imaging (DSI), Q-ball imaging (QBI), Exact QBI (EQBI), spherical deconvolution (SD), generalized diffusion tensor imaging (GDTI), diffusion orientation transform (DOT), etc. The major drawback to these approaches is that they do not consider each fiber independently. They provide information about the major fiber directions but are limited to connectivity assessment.
In contrast, multi-fiber models consider a mixture of independent fibers in each voxel. They provide information about individual fiber bundles underlying characteristics and about the fibers mixing proportions in addition to the fibers orientation. Multi-tensor approaches [2] are an attractive model. Since an individual fiber is well represented by a single tensor, voxels consisting of multiple fibers are expected to be well represented by a mixture of tensors. This modeling enables the computation of diffusion parameters for each fiber independently which is of central interest for fiber integrity assessment. Until recently multi-tensor models were however known to be numerically challenging and unstable, limiting their practical application. In [3] it was theoretically demonstrated that the reason lies in a collinearity of the parameters: only the tensor orientation can be estimated when using a single non-zero b-value. Multiple non-zero b-values are required to estimate the full multi-tensor model and determine simultaneously the tensors direction, the tensors size and the fraction of occupancy.

In this work we propose a novel two-tensor fitting algorithm combined with a novel acquisition scheme which has not been used before for complex fiber structure assessment. Our fitting procedure is formulated in a variational log-Euclidean framework. It ensures non-degenerate tensors and incorporates a novel regularization scheme. Our acquisition scheme combines a single-shell HARDI with the gradients lying on the enclosing cube. It enables the acquisition of multiple non-zero b-values without increasing the minimum achievable echo time (TE) for the single-shell HARDI. In consequence, and contrary to multi-shell HARDI, it does not increase the acquisition time nor the geometric distortion. We evaluate our resulting novel strategy CUSP-MFM (CUsbe+SPhere Multi-Fiber Model) via various experiments with both synthetic and real data experiments with short duration acquisitions. We report qualitative and quantitative fitting performances as well as tractography results. It shows the ability of CUSP-MFM of assessing complex fiber structures with clinically compatible acquisition time. 


\section{MATERIAL AND METHODS.}

\subsection{A variational log-Euclidean two-tensor fitting proce- dure.}

We consider each voxel to be composed of three compartments: two anisotropic compartments representing two fibers and one isotropic compartment modeling the diffusion of free water. The DW signal $S_{k}$ along a gradient direction $\mathbf{g}_{k}$ is then modeled as the following Gaussian mixture [2]:

$S_{k}(\mathbf{D}, \mathbf{f})=S_{0}\left(f_{0} e^{-b_{k} D_{\text {iso }}}+f_{1} e^{-b_{k} \mathbf{g}_{k}^{T} \mathbf{D}_{1} \mathbf{g}_{k}}+f_{2} e^{-b_{k} \mathbf{g}_{k}^{T} \mathbf{D}_{2} \mathbf{g}_{k}}\right)$,

where $D_{\text {iso }}$ is the diffusivity of free water, $b_{k}$ is the applied b-value for the gradient direction $k, \mathbf{D}=\left(\mathbf{D}_{1}, \mathbf{D}_{2}\right)$ are two tensors representing two fibers and $\mathbf{f}=\left(f_{0}, f_{1}, f_{2}\right)$ are the fractions of occupancy of each compartment $\left(\sum_{j=0}^{2} f_{j}=1\right)$.

To ensure the symmetric definite positive property of each tensor we parameterize them in the log-Euclidean framework by setting $\mathbf{L}=\left(\mathbf{L}_{1}, \mathbf{L}_{2}\right)=\left(\log \left(\mathbf{D}_{1}\right), \log \left(\mathbf{D}_{2}\right)\right)$. The simultaneous estimation and regularization of $\mathbf{f}$ and $\mathbf{L}$ (and consequently $\mathbf{D}$ ) is performed via a variational formulation. We consider the image domain $\Omega$ to be a regular 3-dimensional (3D) grid and minimize the following energy:

$$
(\widehat{\mathbf{L}}, \widehat{\mathbf{f}})=\arg \min _{\mathbf{L}, \mathbf{f}} \int_{\mathbf{x} \in \Omega} U_{\text {data }}(\mathbf{L}, \mathbf{f}, \mathbf{x})+\alpha U_{\text {reg }}(\mathbf{L}, \mathbf{f}, \mathbf{x}) \mathrm{d} \mathbf{x},
$$

The data-attachment term $U_{\text {data }}$ is given by a classical leastsquare criteria:

$$
U_{\text {data }}(\mathbf{L}, \mathbf{f}, \mathbf{x})=\sum_{k=1}^{N_{g}}\left[S_{k}\left(e^{\mathbf{L}(\mathbf{x})}, \mathbf{f}(\mathbf{x})\right)-\mathbf{y}_{k}(\mathbf{x})\right]^{2},
$$

where $\mathbf{y}_{k}$ is the measured image for the gradient direction $k$ and $N_{g}$ is the number of gradients directions. The term $U_{\text {reg }}$ enables us to regularize the tensors estimation with a model that exploits spatial homogeneity. We consider an anisotropic regularization model to preserve sharp contours and separate the regularization for each tensor. We consider the widely used minimization of $\phi$-functionals of the spatial gradient's norm $\left\|\nabla \mathbf{L}_{j}(\mathbf{x})\right\|$ and minimize the following energy:

$$
U_{\mathrm{reg}}(\mathbf{L}, \mathbf{f}, \mathbf{x})=\sum_{j=1}^{2} \int_{\mathbf{x} \in \Omega} \phi\left(\left\|\nabla \mathbf{L}_{j}(\mathbf{x})\right\|\right) \mathrm{d} \mathbf{x},
$$

with $\phi(s)=\sqrt{1+s^{2} / K^{2}}$ which accounts for anisotropic regularization, $K$ being a normalization factor for the gradient. Following the one-tensor log-Euclidean model of [4] we set $\left\|\nabla \mathbf{L}_{j}(\mathbf{x})\right\|^{2}=\sum_{m=1}^{3}\left\|\partial_{j, m} \mathbf{L}(\mathbf{x})\right\|_{\text {LE }}^{2}$ with $\|\cdot\|_{\mathrm{LE}}$ the log-Euclidean metric. The partial derivatives $\partial_{j, m} \mathbf{L}(\mathbf{x})$ are approximated with a finite difference scheme. We take into account a special property of multi-fiber models by relating neighboring tensors which are part of the same fiber tract. This is achieved by considering the two most similar neighbors $\mathbf{L}_{q}\left(\mathbf{x} \pm \mathbf{x}_{m}\right)$ to $\mathbf{L}_{j}(\mathbf{x})$ when computing the finite difference in a direction $\mathbf{x}_{m}(m \in\{1,2,3\})$ :

$$
\begin{aligned}
\partial_{j, m} \mathbf{L}(\mathbf{x}) \approx & \left(\arg \min _{\mathbf{L}_{q}}\left\|\mathbf{L}_{q}\left(\mathbf{x}+\mathbf{x}_{m}\right)-\mathbf{L}_{j}(\mathbf{x})\right\|_{\mathrm{LE}}-2 \mathbf{L}_{j}(\mathbf{x})\right. \\
& \left.+\arg \min _{\mathbf{L}_{q}}\left\|\mathbf{L}_{q}\left(\mathbf{x}-\mathbf{x}_{m}\right)-\mathbf{L}_{j}(\mathbf{x})\right\|_{\mathrm{LE}}\right) /\left(2\left\|\mathbf{x}_{m}\right\|\right)
\end{aligned}
$$

We parameterize each tensor by its eigenvalues $\Lambda_{j}=$ $\operatorname{diag}\left(\lambda_{j 1}, \lambda_{j 2}, \lambda_{j 3}\right)\left(\lambda_{j 1} \geq \lambda_{j 2} \geq \lambda_{j 3}\right)$ and the Euler angles $\left(\theta_{j}, \psi_{j}, \phi_{j}\right)$. This representation enables efficient optimization of the parameters, as well as enabling the choice of introducing further constraints on the estimated tensors: symmetry of the eigenvalues, cylindrical shape of each tensor, bounds on the magnitude of the eigenvalues, equiplanar tensors, etc.

The MFM parameters are estimated by performing an iterative minimization which requires an initial guess. We consider the one-tensor solution $\mathbf{D}_{1 \mathrm{~T}}$ and initialize $\exp \mathbf{L}_{1}^{(0)}$ and $\exp \mathbf{L}_{2}^{(0)}$ according to a rotation of $\mathbf{D}_{1 \mathrm{~T}}$ of angle $\varphi= \pm \frac{\lambda_{2}^{1 \mathrm{~T}}}{\lambda_{1}^{1 \mathrm{~T}}} \frac{\pi}{4}$ in the plane formed by $\left(\lambda_{1}^{1 \mathrm{~T}}, \lambda_{2}^{1 \mathrm{~T}}\right)$ the two largest eigen values of $\mathbf{D}_{1 \mathrm{~T}}$. When $\lambda_{1}^{1 \mathrm{~T}}>>\lambda_{2}^{1 \mathrm{~T}}$, which likely indicates an individual fiber bundle in that voxel, we have $\varphi \approx 0$ and the initial $\mathbf{D}_{j}$ 's are two tensors with almost parallel principal diffusivities. In contrast, when $\lambda_{1}^{1 \mathrm{~T}}=\lambda_{2}^{1 \mathrm{~T}}$, the initial $\mathbf{D}_{j}$ 's describe two tensors whose principal diffusivities are perpendicular.

The minimization is performed using the BOBYQA algorithm [5], a well-established iterative derivative-free boundconstrained optimization technique using quadratic approximations for the objective function at each iteration. It converges quickly and enables the introduction of constraints such as $f_{j} \in[0,1]$. We found BOBYQA to be less sensitive to local minima than a conjugate gradient descent scheme.

\subsection{The CUSP gradient encoding scheme.}

As theoretically demonstrated in [3] multiple non-zero bvalues are required to fully estimate two-tensor models. Using separate single-shell HARDI at different b-values leads to different TE for each shell. It leads to different geometric distortion patterns and is subject to spatial misregistration caused by patient motions between scans. Multi-shell HARDI as used in [3] combines several shells in a unique encoding scheme. It however imposes to use the minimum TE achievable for the largest b-value, which is suboptimal for the low b-value measurements. The larger TE also leads to significantly increased geometric and intensity distortions.

We propose to employ one single-shell HARDI combined with the hexa- and tetrahedral gradients which lie on the edges and the corners of the enclosing cube. The six hexahedral gradients $\{(1,1,0),(0,1,1), \ldots\}$ are $\sqrt{2}$-norm gradients and double the nominal $b$-value ${ }^{1}$. The four tetrahedral $\{(1,1,1),(-1,1,1), \ldots\}$ are $\sqrt{3}$-norm gradients and provide b-values three times larger the nominal b-value. Further $b$-values can be acquired by selecting gradient directions which have a squared norm ranging between 0 and 3 . To our knowledge, this is the first report of utilizing this type of acquisition to enable the estimation of a multiple fiber model. Employing such an encoding scheme enables the acquisition of multiple high b-values while using the same TE than a regular single-shell HARDI. It enables the full two-tensor model

\footnotetext{
${ }^{1}$ The applied b-value $b_{k}$ is related to the nominal b-value $b_{\text {nominal }}$ and the gradient norm $\left\|g_{k}\right\|$ by the relation $: b_{k}=b_{\text {nominal }}\left\|g_{k}\right\|^{2}$.
} 


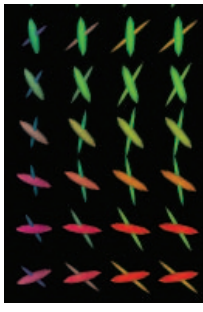

(a)

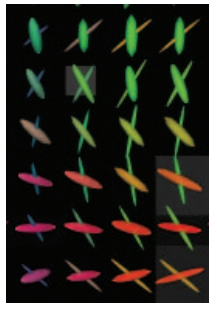

(b)

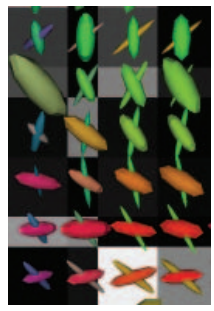

(c)
Fig. 1. (a) One hundred synthetic tensors (cropped) crossing with the same angle $\left(50^{\circ}\right)$. Estimated tensors with CUSP35-MFM (b) and HARDI35-MFM (c) superimposed on the estimated fraction $f_{1}$. It qualitatively shows better results when using CUSP35-MFM.

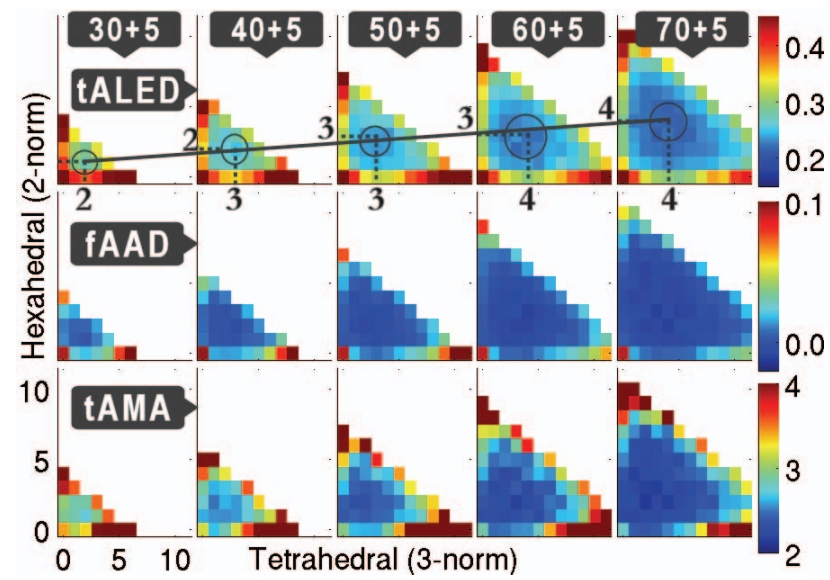

Fig. 2. Assessment of the optimal number of repetitions (0 to 10) for the hexa- and tetrahedral gradients for various total number of images $(5 b=0)$. Each pixel shows the mean of the metric over 800 tensors ( 8 crossing angles from 20 to $90^{\circ}, 100$ configurations each).

estimation without any increase of the acquisition time and of the geometric and intensity distortion.

\section{RESULTS}

CUSP-MFM estimator was parallelized in space and implemented in $\mathrm{C}++$. The model parameters were set as follows: $D_{\text {iso }}=3.010^{-3} \mathrm{~mm}^{2} / \mathrm{s}, \mathrm{K}=0.01, \alpha=2$. The fractions were initialized to $\mathbf{f}^{(0)}(\cdot)=(0.1,0.45,0.45)$. The tensors were constrained to have a cylindrical shape $\left(\lambda_{j, 2}=\lambda_{j, 3}\right.$ for $\left.j=1,2\right)$.

We first assessed CUSP-MFM performances with synthetic experiments. Consistently to real data a trace of $2.110^{-3} \mathrm{~mm}^{2} / \mathrm{s}$ and varying $\mathrm{FA}\left(\mathrm{FA}_{1}=0.9 ; \mathrm{FA}_{2}=0.7\right)$ were considered for the tensor profile representing an individual fiber bundle. The fractions were set to $\left(f_{0}, f_{1}, f_{2}\right)$ $=(0.15,0.6,0.25)$. The DW signal was simulated with $b_{\text {nominal }}$ $=1000 \mathrm{~s} / \mathrm{mm}^{2}$ for different acquisition schemes and corrupted by a Rician noise (SNR of $30 \mathrm{~dB}$ for the $b=0$ images). The estimated tensors were compared to the reference standard by considering the average minimum angle (tAMA [2]) and the average log-euclidean distance (tALED) which takes into

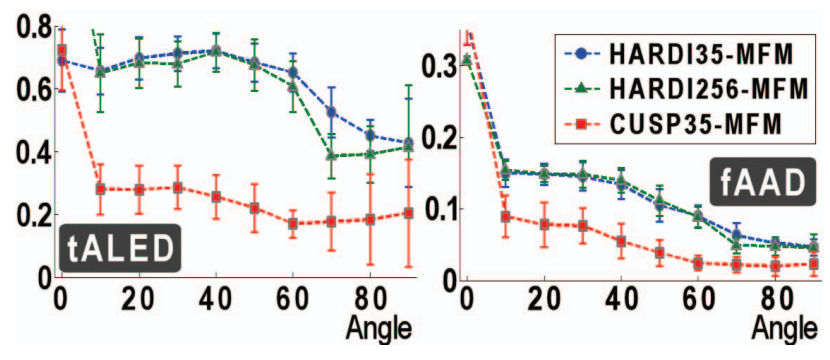

Fig. 3. Mean and variance of the tALED/fAAD metrics over the one hundred synthetic tensors for various crossing angles. It shows the quantitative improvement when using CUSP35-MFM.

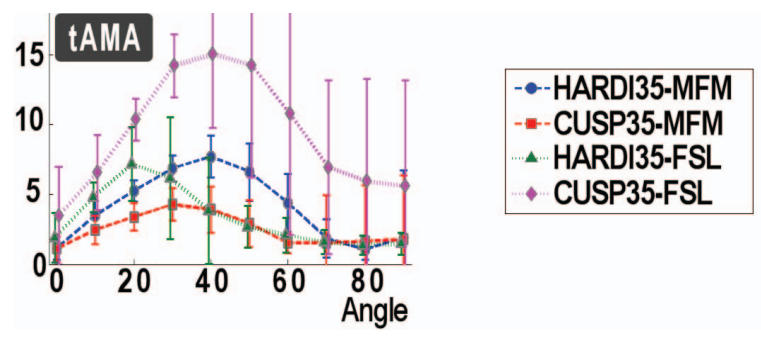

Fig. 4. Mean and variance of the tAMA metric for various crossing angles and estimation strategies. CUSP35-MFM has the best angular resolution, especially for small angles.

account a possible permutation between the tensors:

$$
\begin{array}{r}
\operatorname{tALED}\left(D^{\mathrm{a}}, D^{\mathrm{b}}\right)=\min \left(\left\|D_{1}^{\mathrm{a}}-D_{1}^{\mathrm{b}}\right\|_{\mathrm{LE}}+\left\|D_{2}^{\mathrm{a}}-D_{2}^{\mathrm{b}}\right\|_{\mathrm{LE}},\right. \\
\left.\left\|D_{1}^{\mathrm{a}}-D_{2}^{\mathrm{b}}\right\|_{\mathrm{LE}}+\left\|D_{2}^{\mathrm{a}}-D_{1}^{\mathrm{b}}\right\|_{\mathrm{LE}}\right) .
\end{array}
$$

The tALED metric enables to fully compare tensors and not solely the main direction as with tAMA. The fractions were compared in terms of average absolute difference (fAAD). We generated a set of phantoms containing one hundred twotensor models crossing with a given angle in various configurations (see Fig. 1a). We first assessed how many repetitions of the hexa- and tetrahedral gradients were necessary to counterbalance the lower SNR associated with these high b-value measurements. Fig. 2 suggests an approximately linear relationship between the optimal numbers of repetitions and $N_{g}$ the number of $b \neq 0$ images: \#hexa $=\left\lfloor 0.07 N_{g}-\right.$ $0.9\rfloor$, \#tetra $=\left\lfloor 0.05 N_{g}-0.7\right\rfloor$. In the following we focused on very short duration acquisitions which are of great interest for clinical applications. As suggested by Fig. 2 we considered CUSP35 ( $5 \mathrm{x} b=0$, one shell 16directions, $1 \mathrm{x}$ hexahedral, $2 \mathrm{x}$ tetrahedral) and compared it to a regular single-shell HARDI35 ( $5 \mathrm{x} b=0$, one shell $30 d$.). Fig. 1b-c qualitatively shows the improvement when using CUSP35 instead of HARDI35. Fig. 3 quantitatively reports the estimation accuracy with the tALED and fAAD metrics. Particularly, it shows that employing a large number of directions $(5 \mathrm{x} b=0,251 d$.) does not dramatically improve the results (HARDI256), whereas introducing multiple non-zero b-values does (CUSP35). Fig. 4 compares our approach to the ball-and-stick model of FSL. It shows CUSP35-MFM to achieve in average the best angular resolution, particularly for small angles, while it provides more 

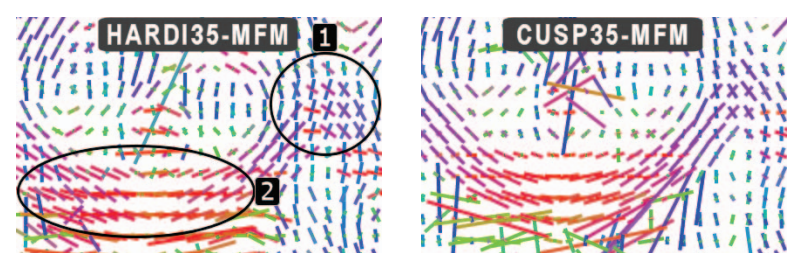

Fig. 5. Tensors estimated on clinical data, showing more realistic configurations with CUSP35-MFM (see areas 1 and 2).

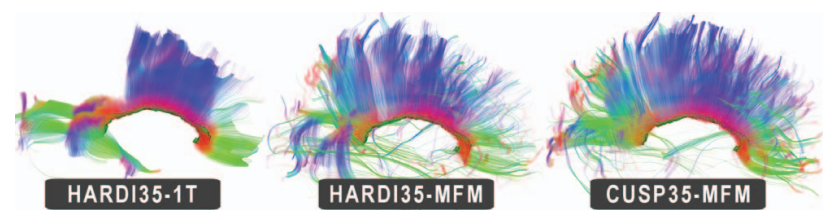

Fig. 6. Tractography results (no tensor model selection applied before generating the tracts, no fiber selection applied).

information for clinical studies by estimating the full tensors.

We also evaluated CUSP-MFM on clinical data (Siemens 3T Trio, 32 channel head coil, 66 slices, FOV $=215 \mathrm{~mm}$, matrix $=120$ $\mathrm{x} 120$, resolution $=1.8 \mathrm{x} 1.8 \times 2.4 \mathrm{~mm}^{3}, \mathrm{TE}=86 \mathrm{~ms} / \mathrm{TR}=9500 \mathrm{~ms}$ for both CUSP35 and HARDI35). The CUSP acquisition time was less than 6 minutes and the MFM estimation time $1 \mathrm{~h} 30$ on a 8 Cores $3 \mathrm{Ghz}$ Intel Xeon. The DW-images and gradients were corrected for head motions via affine registration. Fig. 5 shows that CUSP35-MFM provides 1) a better tensor uniformity and better crossing fibers (area 1) and 2) a better alignment of the two tensors in the single fiber region of the corpus callosum (CC) (area 2). We implemented a multi-fiber subvoxel tractography algorithm which at each step selects the most aligned tensor with the current fiber direction. Fig. 6 reports the tractography results performed from a seeding region of three slices drawn in the inter-hemispheric part of the CC. First it shows the benefits of employing a multi-fiber model rather than a one-tensor (1T) model. Second it shows better results when using CUSP35-MFM instead of HARDI35-MFM. CUSP-MFM leads to 1) a smaller amount of fibers outside of the $\mathrm{CC}$ and 2) a higher density of fibers in the frontal and occipital lobes.

\section{DISCUSSION}

The major drawback to DSI, QBI, EQBI, DOT, SD or GDTI is that they do not consider each fiber tract independently and do not enable the assessment of individual fiber bundle properties which is of central interest for many clinical studies. Generalization of scalar diffusion parameters such as the generalized fractional anisotropy (GFA) can be computed but represents a DW signal dispersion property rather than a fiber property. For example the GFA of two uniform crossing fibers has been shown to decrease in the crossing region, which is not clinically relevant. Two uniform crossing fibers should have constant diffusion scalar parameters along their path.

Multi-fiber models enable to perform both tractography and individual fiber bundles' characteristics assessment. We have proposed a novel multi-fiber assessment strategy CUSPMFM which relies on both a novel multi-tensor fitting procedure (MFM) and a novel acquisition scheme (CUSP). CUSP satisfies the need of multiple non-zero b-values without increasing the TE and in consequence without any impact on the acquisition time and on the geometric and intensity distortion. We experimentally observed CUSP-MFM to achieve a better tensor angular resolution than HARDI-MFM (Fig. 4 and 5), whereas in an algebraic point of view only the tensor magnitude and the fractions are collinear with HARDI [3]. However, consistently with the literature, we verify that probing the diffusion signal at higher b-values helps in differentiating the compartments. To overcome the fact that images acquired at higher b-values have a lower SNR, we provided a relation to determine the optimal number of repetitions of the high b-value measurements for a given acquisition length. We currently focused our evaluation on very short duration acquisitions of 35 images. It shows the ability of CUSP35-MFM to recover complex brain fiber structure in a clinically compatible acquisition time. In future work we will investigate an acquisition-based technique to select the number of fibers at each voxel, based on analysing the DW signal at the multiple b-values provided by CUSP. Preliminary results show good results. We will also investigate in more details the optimal parameters for CUSP-MFM. In particular we will assess the selection of the optimal nominal b-value and evaluate the benefits of employing a finer discretization of the cube's edges which will provide a higher number of unique non-zero bvalues.

\section{REFERENCES}

[1] P J Basser, J Mattiello, and D LeBihan, "Estimation of the effective self-diffusion tensor from the NMR spin echo," J Magn Reson B, vol. 103, no. 3, pp. 247-254, 1994.

[2] D S Tuch, T G Reese, M R Wiegell, N Makris, J W Belliveau, and V J Wedeen, "High angular resolution diffusion imaging reveals intravoxel white matter fiber heterogeneity," MRM, vol. 48, no. 4, pp. 577-582, 2002.

[3] B Scherrer and S K Warfield, "Why multiple b-values are required for multi-tensor models: evaluation with a constrained log-Euclidean model," in ISBI 2010, Rotterdam, Netherlands, 2010, pp. 1389-1392, IEEE Press.

[4] P Fillard, X Pennec, V Arsigny, and N Ayache, "Clinical DT-MRI estimation, smoothing, and fiber tracking with logEuclidean metrics," IEEE Trans Med Imaging, vol. 26, no. 11, pp. 1472-1482, 2007.

[5] M J D Powell, "The BOBYQA algorithm for bound constrained optimization without derivatives," in Technical report NA2009/06, Department of Applied Mathematics and Theoretical Physics, Cambridge England, 2009.

[6] S Peled, S Whalen, F A Jolesz, and A J Golby, "High b-value apparent diffusion-weighted images from curve-ball dti," J Magn Reson Imaging, vol. 30, no. 1, pp. 243-248, Jul 2009.

This investigation was supported in part by NIH grants R01 RR021885, R01 EB008015, R03 EB008680, R01 LM010033 and UL1 RR025758. 